\title{
Neural Correlates of Probabilistic Category Learning in Patients with Schizophrenia
}

\author{
Thomas W. Weickert, ${ }^{1,2}$ Terry E. Goldberg, ${ }^{1,3}$ Joseph H. Callicott, ${ }^{1}$ Qiang Chen, ${ }^{1}$ Jose A. Apud, ${ }^{1}$ Sumitra Das, ${ }^{1}$ \\ Brad J. Zoltick, ${ }^{1}$ Michael F. Egan, ${ }^{1}$ Martijn Meeter, ${ }^{4}$ Catherine Myers, ${ }^{5}$ Mark A. Gluck, ${ }^{6}$ Daniel R. Weinberger ${ }^{1}$ and \\ Venkata S. Mattay ${ }^{1}$ \\ ${ }^{1}$ Genes, Cognition and Psychosis Program, Clinical Brain Disorders Branch, National Institute of Mental Health-National Institutes of Health, Bethesda, \\ Maryland 20892, ${ }^{2}$ Prince of Wales Medical Research Institute, University of New South Wales, Randwick, 2031 Australia, ${ }^{3}$ Division of Psychiatry Research, \\ Zucker Hillside Hospital, Glen Oaks, New York 11004, ${ }^{4}$ Department of Cognitive Psychology, Vrije Universiteit, 1081 BT Amsterdam, The Netherlands, and \\ ${ }^{5}$ Department of Psychology and ${ }^{6}$ Center for Molecular and Behavioral Neuroscience, Rutgers University, Newark, New Jersey 07102
}

Functional neuroimaging studies of probabilistic category learning in healthy adults report activation of cortical-striatal circuitry. Based on previous findings of normal learning rate concurrent with an overall performance deficit in patients with schizophrenia, we hypothesized that relative to healthy adults, patients with schizophrenia would display preserved caudate nucleus and abnormal prefrontal cortex activation during probabilistic category learning. Forty patients with schizophrenia receiving antipsychotic medication and 25 healthy participants were assessed on interleaved blocks of probabilistic category learning and control tasks while undergoing blood oxygenation level-dependent functional magnetic resonance imaging. In addition to the whole sample of patients with schizophrenia and healthy adults, a subset of patients and healthy adults matched for good learning was also compared. In the whole sample analysis, patients with schizophrenia displayed impaired performance in conjunction with normal learning rate relative to healthy adults. The matched comparison of patients and healthy adults classified as good learners revealed greater caudate and dorsolateral prefrontal cortex activity in the healthy adults and greater activation in a more rostral region of the dorsolateral prefrontal, cingulate, parahippocampal and parietal cortex in patients. These results demonstrate that successful probabilistic category learning can occur in the absence of normal frontal-striatal function. Based on analyses of the patients and healthy adults matched on learning and performance, a minority of patients with schizophrenia achieve successful probabilistic category learning and performance levels through differential activation of a circumscribed neural network which suggests a compensatory mechanism in patients showing successful learning.

Key words: schizophrenia; prefrontal cortex; caudate; parahippocampus; learning; cognition

\section{Introduction}

Probabilistic category learning involves a gradual learning of cue-outcome associations putatively without conscious knowledge of the probabilistic frequencies determining those associations (Knowlton et al., 1994). Previous studies of impaired probabilistic category learning rate using the "weather prediction" task in patients with striatal dysfunction suggest that this type of nondeclarative learning is related to striatum function (Knowlton et al., 1996a,b). Functional neuroimaging studies examining probabilistic category learning in healthy adults have reliably demonstrated activation of a neural network that includes the caudate nucleus and the prefrontal and parietal cortices (Poldrack et al., 1999, 2001; Fera et al., 2005).

Normal probabilistic category learning rates in patients with schizophrenia (Kéri et al., 2000, 2005; Weickert et al., 2002) are

\footnotetext{
Received Sept. 11, 2008; revised Nov. 27, 2008; accepted Dec. 27, 2008.

This work was supported by the Intramural Research Program of the National Institutes of Health-National Institute of Mental Health.

Correspondence should be addressed to Thomas W. Weickert, Prince of Wales Medical Research Institute, University of New South Wales, Randwick, 2031 Australia. E-mail: t.weickert@unsw.edu.au.

DOI:10.1523/JNEUROSCI.4341-08.2009

Copyright $\odot 2009$ Society for Neuroscience $\quad$ 0270-6474/09/291244-11\$15.00/0
}

suggestive of normal striatal function in these patients, which is consistent with previous studies of probability learning (Johnston and Bursill, 1973) and nondeclarative motor skill learning in patients with schizophrenia (Clare et al., 1993) and nondeclarative learning during normal aging (Howard and Howard, 1989; Mutter and Pliske, 1994). In addition to normal learning rates, studies of probabilistic category learning in patients with schizophrenia have also revealed impaired overall performance levels (Weickert et al., 2002; Kéri et al., 2005) suggestive of dysfunctional prefrontal cortex input (commonly obtained in neuroimaging studies of patients with schizophrenia) (for review, see Tan et al., 2007) to putatively normal caudate activity within the basal ganglia circuitry described by Alexander et al. (1986). However, the large variation in performance typical during the early trials of probabilistic category learning may have obscured differences in whole group analyses of learning rate between patients with schizophrenia and healthy participants (Kéri et al., 2000, 2005; Weickert et al., 2002). In a recent probabilistic category learning study, Foerde et al. (2008) obtained impaired learning rate and performance in patients with schizophrenia relative to healthy adults, suggesting abnormal caudate function in schizophrenia. However, in the Foerde et al. (2008) 
a.

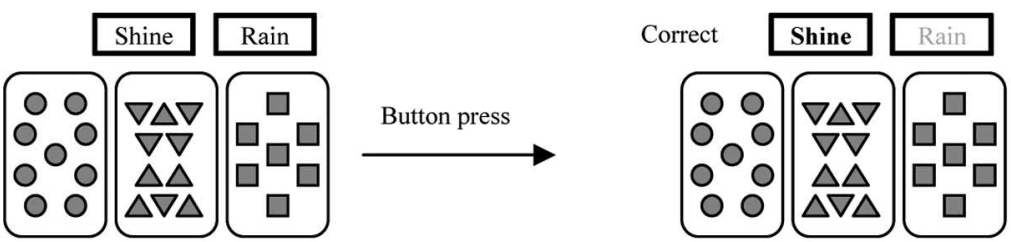

b.

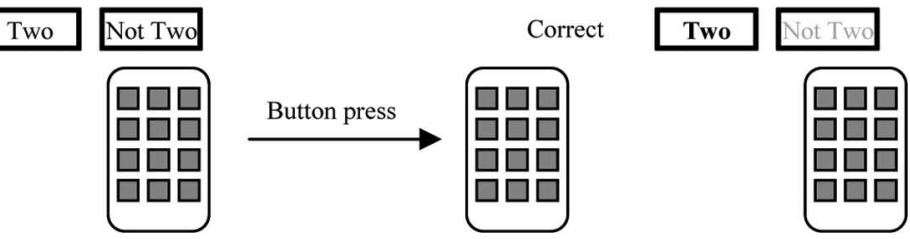

Figure 1. $\boldsymbol{a}, \boldsymbol{b}$, Example of probabilistic category learning $(\boldsymbol{a})$ and perceptual-motor control $(\boldsymbol{b})$ trials.

Table 1. Probability structure of probabilistic learning (weather prediction) task

\begin{tabular}{|c|c|c|c|c|c|c|}
\hline \multirow[b]{2}{*}{ Cue pattern } & \multicolumn{4}{|c|}{ Cue } & \multirow[b]{2}{*}{$p$ (cue combination) } & \multirow[b]{2}{*}{$p$ (outcome) } \\
\hline & 1 & 2 & 3 & 4 & & \\
\hline 1 & 0 & 0 & 0 & 1 & 0.133 & 0.150 \\
\hline 2 & 0 & 0 & 1 & 0 & 0.087 & 0.385 \\
\hline 3 & 0 & 0 & 1 & 1 & 0.080 & 0.083 \\
\hline 4 & 0 & 1 & 0 & 0 & 0.087 & 0.615 \\
\hline 5 & 0 & 1 & 0 & 1 & 0.067 & 0.200 \\
\hline 6 & 0 & 1 & 1 & 0 & 0.040 & 0.500 \\
\hline 7 & 0 & 1 & 1 & 1 & 0.047 & 0.143 \\
\hline 8 & 1 & 0 & 0 & 0 & 0.133 & 0.850 \\
\hline 9 & 1 & 0 & 0 & 1 & 0.067 & 0.500 \\
\hline 10 & 1 & 0 & 1 & 0 & 0.067 & 0.800 \\
\hline 11 & 1 & 0 & 1 & 1 & 0.033 & 0.400 \\
\hline 12 & 1 & 1 & 0 & 0 & 0.080 & 0.917 \\
\hline 13 & 1 & 1 & 0 & 1 & 0.033 & 0.600 \\
\hline 14 & 1 & 1 & 1 & 0 & 0.047 & 0.857 \\
\hline
\end{tabular}

For any given trial, 1 of the 14 possible cue pattern combinations displayed above appeared on the computer screen with a probability indicated as $p$ (cue combination). As shown above, the probability of the cue combinations to predict"sunshine" (outcome 1) was set at $p$ (outcome). Conversely, the probability of the above cue combinations to predict "rain" (or outcome 2) was equal to $1-p$.

study the probabilistic category learning task differed slightly from previous studies of probabilistic category learning in patients with schizophrenia to the extent that the cue-outcome probabilities were slightly easier to learn, their analysis was based on blocks of 50 trials, and the sample size was small.

In the present study, probabilistic category learning was assessed in patients with schizophrenia and healthy adults during functional magnetic resonance imaging (fMRI) to determine the neural correlates of probabilistic category learning in schizophrenia and the potential relationship between prefrontal cortex function and overall performance level. On the basis of previous studies showing normal acquisition rate in conjunction with impaired overall performance levels during probabilistic category learning in patients with schizophrenia, the hypothesis was that relative to healthy adults, patients with schizophrenia would display normal caudate nucleus and abnormal dorsolateral prefrontal cortex activation during probabilistic category learning. This study appears to be one of the first to examine caudate function in patients with schizophrenia using functional imaging of a cognitive task sensitive to caudate function which reliably elicits caudate activation in healthy adults.

\section{Materials and Methods}

Participants

Forty patients with schizophrenia and 25 healthy participants entered the study. Nine patients and no healthy participants were excluded because of excessive motion during the scanning procedure. Therefore, 31 patients, 23 males and $8 \mathrm{fe}$ males (90\% right-hand dominant) with a diagnosis of schizophrenia (22 inpatients and 9 outpatients) completed this study. Two boardcertified psychiatrists concurred on diagnosis by Structured Clinical Interview for the Diagnostic and Statistical Manual, fourth edition without knowledge of cognitive abilities. The frequency of diagnostic subtypes for these 31 patients was as follows: 16 undifferentiated, 6 paranoid, 3 disorganized, 1 simple, 2 schizoaffective depressed, 2 schizoaffective bipolar, and 1 psychosis not otherwise specified, schizotypal personality disorder. Patients having a history of current substance abuse, head injuries with concomitant loss of consciousness, seizures, CNS infection, diabetes, or hypertension were excluded. Patients were all receiving doses of second generation antipsychotic medication (such as olanzapine and risperidone) at the time of testing with the exception of one patient who refused medication. The mean chlorpromazine equivalent dose for 25 patients was 347.7 $\mathrm{mg}, \mathrm{SD}=287.5 \mathrm{mg}$. Psychotic symptoms assessed in 18 patients using the Positive And Negative Symptom Scale (PANSS) (Kay et al., 1987) produced a mean positive symptom score of $15.0, \mathrm{SD}=3.0$, and a mean negative symptom score of $16.8, \mathrm{SD}=4.5$.

In addition to patients with schizophrenia, 25 healthy participants, 11 males and 14 females (88\% right-hand dominant) recruited through the National Institutes of Health normal volunteer office, completed this study. Healthy participants with a history of psychiatric disorders, current substance abuse, head injuries with concomitant loss of consciousness, seizures, CNS infection, diabetes, or hypertension were excluded. All participants provided informed written consent before participation in this study. The Institutional Review Board of the National Institute of Mental Health provided approval for this study. The mean years of age was not significantly different between patients $($ mean $=29.8, \mathrm{SD}=9.4)$ and healthy participants $($ mean $=29.3, \mathrm{SD}=$ 9.8), $t_{(50)}=0.20, p=0.84$. However, the mean years of education did differ significantly between patients (mean $=13.5, \mathrm{SD}=2.1$ ) and healthy participants $($ mean $=15.7, \mathrm{SD}=1.5), t_{(42)}=3.93, p<0.001$, which was expected on the basis of previous studies comparing patients with schizophrenia to healthy participants. For all participants who had visual refractive abnormalities, corrections were achieved with prescription contact lenses or MRI compatible plastic lenses in a plastic frame.

\section{Probabilistic category learning test}

A version of the probabilistic category learning "weather prediction" task (Poldrack et al., 1999) allowed alternation of the experimental weather prediction task with a perceptual-motor control task. Before entering the scanner all participants were given instructions for both tasks. For the weather prediction task, participants were told that they should make a decision to predict rain or shine on the basis of four distinct cue cards that would be presented either individually or in combinations of up to three cards. They were further told that they should guess at first but gradually they would improve at determining which cue card combinations predict rain or shine. In this probabilistic category learning task participants learn the relationship between two equally occurring outcome variables (rain or shine) and combinations of four cue cards each composed of simple geometric shapes (Fig. 1a). The relationship between cue cards and outcome variables was predetermined on a probabilistic basis (see Table 1 for the cue-outcome probability schedule) and presentations 


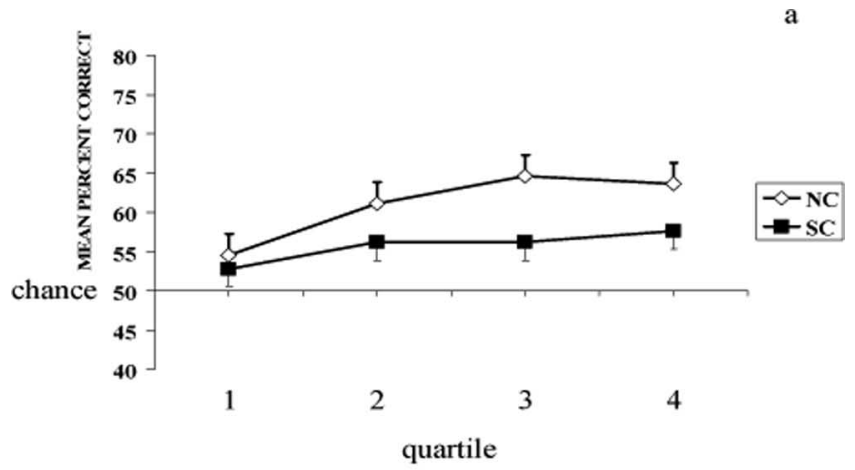

b

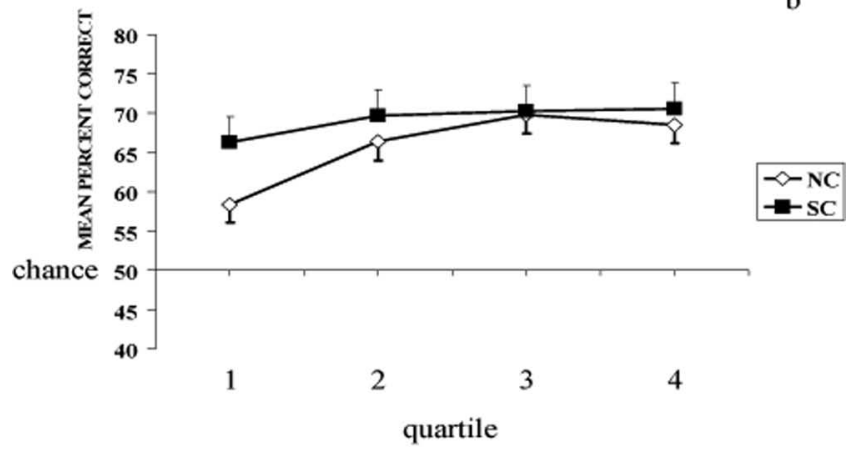

c

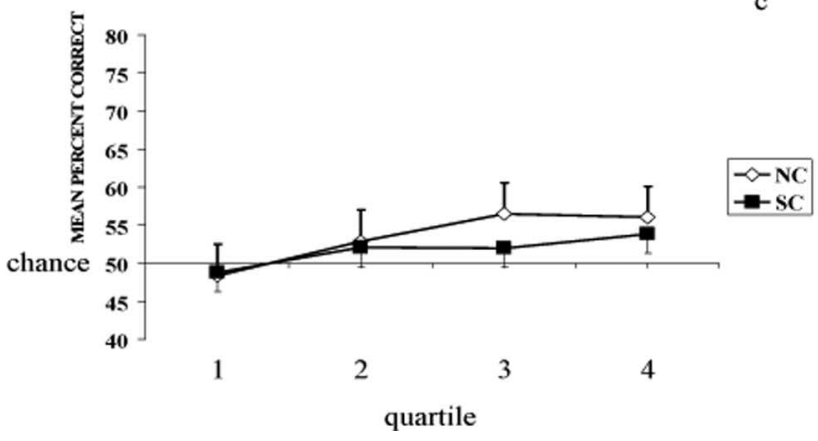

Figure 2. $\boldsymbol{a}-\boldsymbol{c}$, Cumulative percentage correct at each trial block during probabilistic category learning for whole sample of patients with schizophrenia (SC) and healthy participants (NC) (a), those patients with schizophrenia and healthy participants classified as good learners (b), and those patients with schizophrenia and healthy participants classified as poor learners (c).

were randomized with the constraint that identical cue combinations would not appear consecutively and each outcome (rain or shine) was limited to five consecutive occurrences. For the perceptual-motor control task all participants were instructed to determine whether or not two of four identical cue cards were presented during each trial, with the cue cards being presented either individually or in combinations of up to three cards (see Fig. $1 b$ for the perceptual-motor control task stimuli). Presentations of the perceptual-motor control stimuli were randomized with the constraint that identical spatial positions of the four cards did not appear consecutively and each outcome (two or not two) was limited to five consecutive occurrences. Stimuli for both tasks (weather prediction and perceptual-motor control) were presented via a back-projection system and behavioral responses, left (for "shine" or "two") or right (for "rain" or "not two") button presses with the right thumb, were recorded via a fiber-optic response box (Current Designs). All stimuli were displayed on the screen for $4.5 \mathrm{~s}$ with an intertrial interval of $0.5 \mathrm{~s}$. After each response for both experimental and perceptual-motor control tasks, the words "correct" or "incorrect" appeared as feedback to the participant and missed trials were not included in the scoring.

\section{Scanning procedure}

Using a quadrature head coil transceiver, the fMRI study was performed on a 1.5 T magnetic resonance system (General Electric) equipped with gradients capable of generating gradient fields of $40 \mathrm{mT} / \mathrm{m}$ with a slewrate of $180 \mathrm{~T} / \mathrm{m} / \mathrm{s}$. For the blood oxygenation level-dependent (BOLD) fMRI experiment, a gradient echo echoplanar imaging sequence was used, that acquired single-shot images with a matrix size of $64 \times 64$ over a field of view of $200 \times 200 \mathrm{~mm}^{2}$, for a nominal in-plane resolution of $3.125 \times 3.125 \mathrm{~mm}^{2}$. Thirty axial slices with $3 \mathrm{~mm}$ thickness, $1 \mathrm{~mm}$ gap, were oriented inferior to superior, to cover the whole brain. Repetition time, echo time, and flip angle were $3.0 \mathrm{~s}, 50 \mathrm{~ms}$, and $90^{\circ}$, respectively. The experimental paradigm consisted of a simple box-car design with eight cycles during which $30 \mathrm{~s}$ blocks of probabilistic category learning stimuli ( 6 trials/block) alternated with $30 \mathrm{~s}$ blocks of perceptual-motor control task stimuli ( 6 trials/block). Each participant received two runs with each run consisting of 48 weather prediction and 48 perceptualmotor control trials. One hundred-sixty scans were collected in a total scan time of 8 min per fMRI run, giving a total of 96 trials for each task. Task order (probabilistic category learning or perceptual-motor control) was counterbalanced across participants and gender.

\section{Data analysis}

Behavioral data. Percentage correct and reaction times were used as measures of learning the cue-outcome associations during probabilistic category learning. Transformed cumulative percent correct scores and reaction times were analyzed using separate two-way repeated measures ANOVAs with patients and healthy participants as the between-subjects variable. Additionally, a separate independent $t$ test was used to determine group differences with respect to trials on which no responses were made. An identical series of analyses was applied to the perceptual-motor control task data.

Strategy analyses. Because previous work (Gluck et al., 2002) suggests that the strategy used to perform this probabilistic category learning task may influence performance, data from the present study were also analyzed blind to group status (patient versus healthy participant) and matching criteria by using an improved version of a strategy clustering analysis (Meeter et al., 2006). The revised strategy clustering analysis assigns blocks of trials from individual participants to strategies on the basis of the response pattern. Assigned strategies were either complex (multicue strategy), in which participants base their response on the whole configuration of cards, simple (singleton strategy), in which participants respond consistently only to one-card patterns, intermediate (single cue strategy) based on the presence/absence of one card in a 2-3 card combination, or optimal in which participants predicted the outcome most often associated with the cue-card combinations. If the response pattern of a participant did not conform to any of the strategies described above, their performance was categorized as "no detectable strategy." However, classification as such does not necessarily imply that a participant did not use a strategy or strategies; instead they may have used idiosyncratic or probabilistic strategies that were not identified, or they may have switched too rapidly between strategies so that no consistent pattern emerged. The strategy clustering analysis described above (performed by M.M.) was conducted blind to group membership (patient versus healthy participants) and classification of learning status (good versus poor learner). The frequency of each strategy used in both the patients with schizophrenia and healthy participants was compared using a $\chi^{2}$ analysis to establish whether the two groups used qualitatively different strategies to solve the probabilistic category learning task. Other strategy variables comparing patients with schizophrenia and healthy participants were the trial at which the first strategy switch occurred, the total number of strategy switches, the number of strategy switches during the first two quartiles, and the number of strategy switches during the last two quartiles (Meeter et al., 2006, 2008).

Imaging data. Using the approach described by Fera et al. (2005), these data were processed off-line on a Linux workstation, using the general linear model of SPM2. Images for each participant were realigned to the 
Table 2. Mean reaction time (in milliseconds) during probabilistic category learning and perceptual-motor control tasks in patients with schizophrenia and healthy participants

\begin{tabular}{lclll}
\hline & Quartile 1 & Quartile 2 & Quartile 3 & Quartile 4 \\
\hline $\begin{array}{l}\text { Probabilistic category learning } \\
\quad \text { Patients with schizophrenia }\end{array}$ & $1553.4(114.6)$ & $1392.9(90.5)$ & $1547.4(126.2)$ & $1673.9(136.8)$ \\
$\quad$ Healthy participants & $1596.2(127.6)$ & $1428.3(100.8)$ & $1467.3(140.5)$ & $1389.6(152.3)$ \\
$\begin{array}{l}\text { Perceptual-motor control } \\
\text { Patients with schizophrenia }\end{array}$ & $1300.0(75.1)$ & $1383.6(118.6)$ & $1291.5(76.0)$ & $1325.4(115.9)$ \\
$\quad$ Healthy participants & $876.5(85.3)$ & $862.1(134.8)$ & $877.3(86.4)$ & $880.1(131.7)$ \\
\hline
\end{tabular}

Values in parentheses are SE.

eighth volume in the time series to correct for head motion, spatially normalized into a standard stereotactic space (Montreal Neurological Institute template) using a 12 parameter affine model, and smoothed to minimize noise and residual differences in gyral anatomy with a Gaussian filter set at $6 \mathrm{~mm}$ full-width at half-maximum. Voxelwise signal intensities were ratio normalized to the whole-brain global mean. Data sets were also screened for high quality (scan stability) as demonstrated by small motion correction $(<2 \mathrm{~mm})$. To better evaluate the temporal dynamics of learning-related BOLD changes, the time series data were divided into four quartiles of 80 scans each, with 24 weather prediction and 24 perceptual-motor control trials in each quartile.

As a first level of analysis, predetermined condition effects at each voxel were calculated using a $t$-statistic, producing a statistical image for the contrast of weather prediction versus perceptual-motor control tasks for each quartile and for each participant. These individual contrast images were then used in a conservative second-level random effects model that accounts for both scan-to-scan and subject-to-subject variability using one-sample $t$ tests for main effects of condition within each quartile $(p<0.0025)$. Second level analyses were constrained by a mask that was created using regions selected a priori based on those regions shown to be relevant to probabilistic category learning from previous studies (Poldrack et al., 1999, 2001; Fera et al., 2005) by summing the main effect of task activation masks for each quartile ( $p<0.005$, uncorrected) from both groups (see supplemental Fig. 1, available at www.jneurosci.org as supplemental material, for a section view of the mask at center point). Statistical thresholds for second level analyses were set at $p<0.005$ uncorrected, minimum cluster size $(k)=3$. Results that survived small volume corrections for multiple comparisons (false discovery rate-corrected, as described by Genovese et al., 2002) within a priori regions of interest selected from Pick Atlas (http://fmri.wfubmc.edu/cms/ software\#PickAtlas) are indicated within Table 3.

For the group $\times$ condition interactions, the whole sample of patients and healthy participants were initially compared. In addition to whole group analysis, analyses were also conducted between patients with schizophrenia and healthy participants who were matched on probabilistic category learning rate and performance levels. Differential brain activation has been shown during error processing relative to response competition, inhibition, or selection (Menon et al., 2001) illustrating the importance of performance matching between patient and control groups to show effects of the disease processes on brain activity (Curtis et al., 2001; Gould et al., 2006; Davis et al., 2008; Eyler et al., 2008). Because the groups in the present study were not matched on performance, a subsequent group $\times$ condition interaction was performed in which patients and healthy adults were compared on the basis of being classified as good learners. To match performance for imaging comparisons, learning was defined on an individual basis as showing an increase in percentage correct across the quartiles (positive difference score between trials 10 and 96) and displaying a sustained performance approximately equal to or $>70 \%$ correct during quartiles three and four. Because the number of patients and healthy adults classified as good learners using the above definition of learning differed (see below) the 8 patients classified as good learners were further matched on gender and age to 8 of the 16 healthy adults classified as good learners. Second level analyses were constrained by a mask that was created by summing the main effect of task activation masks for each quartile ( $p<0.005$, uncorrected) from both groups. By comparing only those patients and healthy adults classified as good learners, the effects of learning differences between groups could be removed to determine regionally distinct activation differences between patient and healthy participants that were related to task processing.

\section{Results \\ Behavior}

Probabilistic category learning in the whole sample of patients and healthy participants Overall, both patients with schizophrenia and healthy adults displayed probabilistic category learning acquisition rates that were not significantly different. Results of a two-way, repeated measures ANOVA for percentage correct by quartile displayed a significant main effect of group, $F_{(1,54)}=$ $3.95, p=0.05$, a significant main effect of quartile, $F_{(3,162)}=7.48$, $p<0.001$, and no significant group $\times$ quartile interaction, $F_{(3,162)}=1.61, p=0.19$. Regarding reaction times, a separate two-way, repeated measures ANOVA displayed no main effect of group, $F_{(1,54)}=0.27, p=0.60$, no main effect of quartile, $F_{(3,162)}$ $=1.16, p=0.33$, and no significant group $\times$ quartile interaction, $F_{(3,162)}=1.39, p=0.25$ (see Fig. $2 a$ for the cumulative percentage correct at each quartile for patients with schizophrenia and healthy participants during probabilistic category learning, and Table 2 for the mean reaction time and SE at each quartile for patients with schizophrenia and healthy participants during probabilistic category learning). Results of a separate independent $t$ test for the number of trials on which no responses were made during probabilistic category learning displayed a significant group difference between patients with schizophrenia $($ mean $=2.3, \mathrm{SD}=2.8)$ and healthy participants $($ mean $=0.8$, $\mathrm{SD}=1.3), t_{(53)}=2.49, p=0.02$. However, the mean total number of omissions for the patients was only $2 \%$ of the total trials. There was a nonsignificant, weak to no correlation between chlorpromazine equivalent dose and probabilistic category learning acquisition rate with $r=-0.17, p=0.43$. There were significant trends toward mild to moderately strong correlations between probabilistic category learning acquisition rate and PANSS negative symptom severity scores with $r=0.40, p=0.10$; and PANSS positive symptom severity scores with $r=0.41, p=0.09$. Thus, as positive and negative symptom scores increase (i.e., patients are more symptomatic), the probabilistic category learning acquisition rate increases (i.e., learning improves). The finding of significant trends toward moderately strong positive relationships between symptom severity and acquisition rate is directly opposed to our finding of no relationship between antipsychotic medication dose in chlorpromazine equivalents and probabilistic category learning acquisition rate and should be a focus of further studies.

\section{Probabilistic category learning in the matched sample}

Using a more stringent definition of learning (positive slope and sustained performance during latter trials) patients and healthy adults were matched on the basis of learning and classified as good or poor learners. Results of a $\chi^{2}$ analysis comparing the number of good and poor learners in each group revealed a significant difference in the number of good learners (healthy participants $N=16$, patients with schizophrenia $N=8$ ) and poor learners (healthy participants $N=9$, patients with schizophrenia $N=23$ ) in each group, $\chi^{2}(1)=8.24, p=0.004$ (see Fig. $2 b$ for the cumulative percentage correct at each quartile for patients with schizophrenia and healthy participants classified as good learners during the probabilistic category learning task, and Fig. $2 c$ for the cumulative percentage correct at each quartile for patients with schizophrenia and healthy participants classified as 
poor learners during the probabilistic category learning task). There were only 5 patients having PANSS negative symptoms scores that were 5 or more points greater than their PANSS positive symptoms scores. Only 20\% negative syndrome patients could be classified as a good learner and only $23 \%$ non-negative syndrome patients could be classified as good learners. Thus, with percentages being approximately equal, there was not an inordinate advantage for one symptom profile over the other with respect to probabilistic category learning.

\section{Perceptual-motor control task}

ANOVA for percentage correct during the perceptual-motor control task displayed a significant main effect of group, $F_{(1,54)}=$ $3.97, p=0.05$, a significant main effect of quartile, $F_{(3,162)}=4.01, p=0.009$, and no significant group $\times$ quartile interaction, $F_{(3,162)}=0.91, p=0.44$. Although there was a significant main effect of group, patients with schizophrenia performed very well on the control task (patient mean percentage correct $=92.5, \mathrm{SD}=1.7$; healthy participant mean percentage correct $=$ $97.7, \mathrm{SD}=1.9$ ) relative to the typical performance of patients with schizophrenia on cognitive tests in general. Importantly, there was no group $\times$ quartile interaction indicating that there were no learning differences with respect to the perceptual motor control task. Results of a separate independent $t$ test for the number of trials on which no responses were made during the perceptual motor control task displayed a significant group difference between patients with schizophrenia $($ mean $=1.0, \mathrm{SD}=1.5)$ and healthy participants (mean $=0.04, \mathrm{SD}=0.2), t_{(53)}=3.20, p=$ 0.002 . However, once again the mean total number of omissions for the patients was very low, $\sim 1 \%$ of the total trials. ANOVA for reaction time during the perceptual-motor control task displayed a significant main effect of group, $F_{(1,53)}=18.75, p<0.001$, no significant main effect of quartile, $F_{(3,159)}=0.08, p=0.97$, and no significant group $\times$ quartile interaction, $F_{(3,159)}=0.16, p=0.93$ (see Table 2 for the mean reaction time and SE at each quartile for patients and healthy participants during the perceptual-motor control task). As is generally the case, the patients with schizophrenia were significantly slower than the healthy participants during the perceptual-motor control task.

\section{Strategy analyses}

In general, patient with schizophrenia and healthy participant good and poor learners used different strategies during probabilistic category learning (Fig. $3 a, b$ ). Whereas patients with schizophrenia and healthy participants classified as good learners primarily changed from using the complex strategy to using the optimal strategy, patients with schizophrenia and healthy participants classified as poor learners primarily used no discernible strategy throughout the four quartiles.

\section{Patients and healthy participants classified as good learners}

Results from the strategy analysis revealed significant differences between the strategies used by the 8 patients with schizophrenia relative to the 16 healthy participants classified as good learners.
Separate $\chi^{2}$ analyses for each quartile displayed significant differences in the number of patients with schizophrenia and healthy participants classified as good learners with respect to the type of strategy used (including no detectable strategy) during quartile 1 $\left(\chi^{2}(4)=47.9, p<0.001\right)$, quartile $2\left(\chi^{2}(4)=15.5, p<0.004\right)$, quartile $3\left(\chi^{2}(4)=25.9, p<0.001\right)$, and quartile $4\left(\chi^{2}(4)=\right.$ $27.1, p<0.001$ ) (see Fig. $3 a$ for the strategies used in each quartile by the patients with schizophrenia and healthy participants classified as good learners). In general, the primary strategy used by the majority of patients with schizophrenia classified as good learners evolved from a singleton strategy during the first quartile to a complex strategy during the second quartile and finally to the optimal strategy during quartiles 3 and 4 (although there was an equal percentage of patients using a complex strategy during the fourth quartile). Conversely, the primary strategy used by the majority of healthy participants classified as good learners evolved from a complex strategy in quartiles one and two to the optimal strategy during quartiles three and four. Paired $t$ tests of other strategy variables comparing those patients with schizophrenia and healthy participants classified as good learners revealed no significant differences with respect to the trial at which the first strategy switch occurred (patients with schizophrenia mean $=17.4, \mathrm{SD}=2.1$; healthy participants mean $=25.3 ; \mathrm{SD}=$ $\left.15.1 ; t_{(22)}=1.46, p=0.16\right)$, the total number of strategy switches (patients with schizophrenia mean $=3.6, \mathrm{SD}=0.7$; healthy participants mean $\left.=3.3 ; \mathrm{SD}=1.0 ; t_{(22)}=0.93, p=0.36\right)$, the number of strategy switches during the first two quartiles (patients with schizophrenia mean $=2.3, \mathrm{SD}=0.7$; healthy participants mean $\left.=2.1 ; \mathrm{SD}=1.0 ; t_{(22)}=0.33, p=0.75\right)$, and the number of strategy switches during the last two quartiles (patients with schizophrenia mean $=1.4, \mathrm{SD}=0.9$; healthy participants mean $\left.=1.1 ; \mathrm{SD}=0.5 ; t_{(22)}=0.87, p=0.39\right)$. 
Additionally, with respect to the 8 patients with schizophrenia matched to 8 healthy adults on the basis of probabilistic category learning rate and performance, gender, and age, there were no significant differences between strategies used during quartile 1 $\left(\chi^{2}(1)=0.01, p<0.92\right)$, quartile $2\left(\chi^{2}(2)=0.61, p<0.74\right)$, quartile $3\left(\chi^{2}(2)=0.29, p<0.87\right)$, and quartile $4\left(\chi^{2}(2)=0.34\right.$, $p<0.84$ ) (see Fig. $3 c$ for the strategies used in each quartile by the 8 patients with schizophrenia matched to 8 healthy participants). There were also no significant differences between the 8 patients with schizophrenia matched to 8 healthy participants on the basis of trial at which the first switch occurred (patients with schizophrenia mean $=17.4, \mathrm{SD}=2.1$; healthy participants mean $=$ $\left.20.6 ; \mathrm{SD}=5.4 ; t_{(14)}=1.59, p=0.13\right)$, total number of switches (patients with schizophrenia mean $=3.6, \mathrm{SD}=0.7$; healthy participants mean $\left.=3.5 ; \mathrm{SD}=0.9 ; t_{(14)}=0.29, p=0.77\right)$, number of switches during the first two quartiles (patients with schizophrenia mean $=2.3, \mathrm{SD}=0.7$; healthy participants mean $=2.4 ; \mathrm{SD}=$ $\left.0.9 ; t_{(14)}=0.30, p=0.76\right)$, and number of switches during the last two quartiles (patients with schizophrenia mean $=1.4, \mathrm{SD}=0.9$; healthy participants mean $=1.1 ; \mathrm{SD}=0.4 ; t_{(14)}=0.72, p=0.48$ ).

\section{Patients and healthy participants classified as poor learners}

Separate $\chi^{2}$ analyses for each quartile displayed significant differences in the number of patients with schizophrenia and healthy participants classified as poor learners with respect to the type of strategy used (including no detectable strategy) during quartile 1 $\left(\chi^{2}(4)=16.4, p<0.003\right)$, quartile $3\left(\chi^{2}(4)=22.0, p<0.001\right)$, and quartile $4\left(\chi^{2}(4)=26.6, p<0.001\right)$, with no significant difference during quartile $2\left(\chi^{2}(4)=5.4, p<0.25\right.$, NS) (see Fig. $3 b$ for the strategies used in each quartile by the patients with schizophrenia and healthy participants classified as poor learners). In general, the majority of patients with schizophrenia and healthy participants classified as poor learners used no detectable strategy during each of the quartiles (although there were significant differences among smaller numbers of patients and healthy participants using other strategies during quartiles one, three, and four). Paired $t$ tests of other strategy variables comparing those patients with schizophrenia and healthy participants classified as poor learners revealed no significant differences with respect to the trial at which the first strategy switch occurred (patients with schizophrenia mean $=54.3, \mathrm{SD}=26.1$; healthy participants mean $\left.=54.0 ; \mathrm{SD}=32.0 ; t_{(30)}=0.02, p=0.98\right)$, the total number of strategy switches (patients with schizophrenia mean $=1.3, \mathrm{SD}=1.3$; healthy participants mean $=1.8 ; \mathrm{SD}=$ $1.9 ; t_{(30)}=0.74, p=0.46$ ), the number of strategy switches during the first two quartiles (patients with schizophrenia mean $=$ $0.8, \mathrm{SD}=1.2$; healthy participants mean $=1.0 ; \mathrm{SD}=1.2 ; t_{(30)}=$ $0.38, p=0.71$ ), and the number of strategy switches during the last two quartiles (patients with schizophrenia mean $=0.5, \mathrm{SD}=$ 0.7 ; healthy participants mean $=0.8 ; \mathrm{SD}=0.8 ; t_{(30)}=0.86, p=$ $0.40)$.

\section{Imaging}

Whole sample: patients versus healthy participants

Consistent with previous findings during probabilistic category learning in healthy participants (Poldrack et al., 1999, 2001; Fera et al., 2005), the spatial distribution of task-related responses elicited bilateral prefrontal cortex (BA 9/10, 45-46/47), supplementary motor area, premotor cortices (BA 6/8), parietal cortices (BA 40), bilateral caudate nucleus and thalamus in healthy participants. At the group level, the main effect of condition across the four quartiles mapped on to similar locations for the whole sample in both groups (Fig. 4). Whole group differences were a) healthy adults

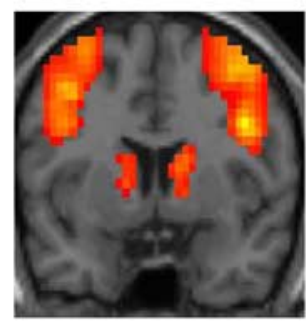

bilateral caudate nucleus

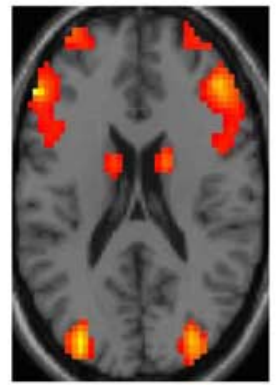

dorsolateral prefrontal cortex

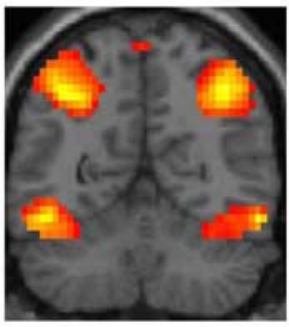

bilateral parietal cortex b) patients with schizophrenia

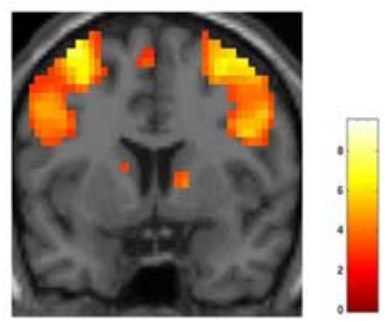

bilateral caudate nucleus

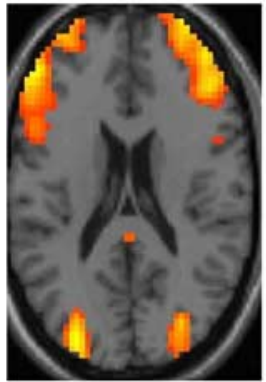

dorsolateral prefrontal cortex

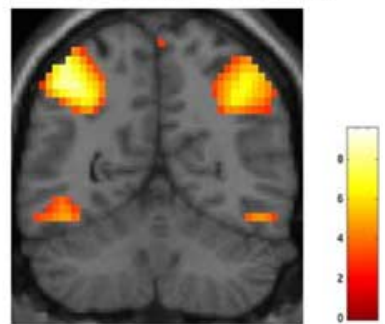

bilateral parietal cortex
Figure 4. $\quad \boldsymbol{a}, \boldsymbol{b}$, Main effects of condition across the four quartiles in healthy adults ( $\boldsymbol{a})$ and patients with schizophrenia $(\boldsymbol{b})$ (extent threshold, $k=3$ voxels, $p<0.005$ uncorrected).

most apparent in the group $\times$ condition interaction analysis: the BOLD response was much greater bilaterally at the level of the dorsolateral prefrontal cortex (BA 9/46) and caudate nucleus in the healthy participants throughout the entire experiment (Fig. $5 a)$. Conversely, the patients with schizophrenia showed a greater BOLD response of a more rostral portion of the left dorsolateral prefrontal cortex (BA 9) and right cingulate gyrus (BA 32) over the four quartiles during probabilistic category learning (Fig. 5b). However, these activation differences may be caused by strategy or performance differences because the groups were not matched on performance.

Matched sample: patients versus healthy participants classified as good learners

The main effect maps for the matched sample also mapped onto similar locations in both groups (Table 3, Fig. 6) with the exception that caudate activation was not obtained and parahippocampal activity was obtained in the eight patients with schizophrenia showing good learning. In the group $\times$ condition interaction analysis for the comparison of 8 patients with schizophrenia and 8 healthy participants classified as good learners, healthy participants displayed greater primarily right-sided activation of prefrontal cortex (BA 9/10), temporal cortex (BA 37), precuneus (BA 7), cuneus (BA 17), and caudate nucleus during quartiles 1 and 2; and prefrontal cortex (BA 9/10), ventral prefrontal cortex (BA 47), temporal cortex (BA 20/37), inferior parietal cortex (BA 40), precuneus (BA 7), cuneus (BA 17) and caudate nucleus during quartiles 3 and 4 relative to the patients with schizophrenia 
(Table 3, Fig. 6a). Relative to the eight healthy participants from the matched comparison, the eight patients with schizophrenia displayed greater primarily right-sided activation of a more rostral region of the dorsolateral prefrontal cortex (BA 9), cingulate (BA 31, 32), temporal cortex (BA 21), and left inferior parietal cortex (BA 40) during quartiles 1 and 2; and a more rostral region of right prefrontal cortex (BA 9), bilateral cingulate (BA $31 / 32$ ), right temporal cortex (BA 21), bilateral parahippocampal gyrus (BA 27/36), and cuneus (BA 18) during quartiles 3 and 4 (Table 3, Fig. 6b).

\section{Discussion}

On the basis of the whole sample of patients with schizophrenia and healthy participants, patients showed a normal probabilistic category learning acquisition rate concurrent with an overall performance deficit relative to healthy participants. These findings support results from previous studies (Weickert et al., 2002; Kéri et al., 2005) of probabilistic category learning in patients with schizophrenia. Although an overall performance deficit in conjunction with a normal learning rate was obtained in the whole sample; in an important departure from previous studies of probabilistic category learning in patients with schizophrenia, the present study showed significant differences in the numbers of patients with schizophrenia and healthy participants who displayed probabilistic category learning (defined as showing both a positive slope and sustained performance during latter trials). The learning criterion was independently confirmed by the blind strategy analysis showing that the majority of patients and healthy participants classified as good learners used various strategies whereas the majority of patients and healthy participants classified as poor learners used no detectable strategy. There were significantly more healthy participants classified as good learners and significantly more patients with schizophrenia classified as poor learners. Previous reward based probabilistic learning studies have also revealed both good and poor learning groups among healthy adult samples (Joel et al., 2005; Schönberg et al., 2007). Differences in the frequencies of good and poor learning patients with schizophrenia and healthy adults may have contributed to the apparent dissociation between learning rate and overall performance observed in the present and previous (Weickert et al., 2002; Kéri et al., 2005) studies by producing a dilution of learning rate differences when combining good and poor learners.

Similar to previous neuroimaging studies of feed back based probabilistic category learning in healthy adults (Poldrack et al., 1999, 2001; Fera et al., 2005), the present study displayed activation of a neural network that included the prefrontal and parietal cortices and the caudate nucleus in healthy participants. The first level of analysis showed activation of the prefrontal cortex and caudate nucleus in both healthy participants and patients with schizophrenia. However, contrast between all patients with schizophrenia and healthy participants revealed greater dorsolateral prefrontal cortex and caudate nucleus activation in the healthy participants relative to patients and greater activation of a more rostral region of dorsolateral prefrontal cortex (BA 9) and cingulate gyrus (BA 32) in patients relative to healthy participants. Insufficient dorsolateral prefrontal cortex and caudate activation in patients also supports previous studies (Pickar et al., 1996; Heinz et al., 1998; Bertolino et al., 1999, 2000; MeyerLindenberg et al., 2002) showing abnormal frontal-striatal function in patients with schizophrenia. Reiss et al. (2006) has also shown a relative lack of striatal activation during a procedural learning serial reaction time task in patients with schizophrenia with no significant difference in reaction time and performance level relative to healthy adults.

Insufficient activation of the caudate nucleus and dorsolateral prefrontal cortex would raise the question of how some patients with schizophrenia successfully achieve probabilistic category 
Table 3. Significant BOLD fMRI responses for interactions between groups during the four quartiles

\begin{tabular}{|c|c|c|c|c|c|c|c|c|c|}
\hline \multicolumn{5}{|c|}{ Quartile 1-2 } & \multicolumn{5}{|c|}{ Quartile 3-4 } \\
\hline \multirow{2}{*}{\multicolumn{3}{|c|}{ Talairach coordinates $(x, y, z)$}} & Z & & & & & & \\
\hline & & & score & Brodmann area & \multicolumn{3}{|c|}{ Talairach coordinates $(x, y, z)$} & Zscore & Brodmann area \\
\hline \multicolumn{10}{|c|}{ Healthy participants $>$ Patients with schizophrenia } \\
\hline-31 & 51 & -6 & 3.85 & BA $10^{* *}$ & -34 & 54 & -6 & 4.54 & $\mathrm{BA} 10^{* * *}$ \\
\hline \multirow[t]{2}{*}{+44} & 14 & 36 & 2.98 & BA 9 & +52 & 14 & 32 & 3.21 & BA 9 \\
\hline & & & & & -41 & 24 & -8 & 4.69 & BA $47^{* * *}$ \\
\hline+3 & 27 & 50 & 3.09 & BA 8 & -3 & 30 & 46 & 3.19 & $\mathrm{BA} 8^{*}$ \\
\hline \multirow[t]{2}{*}{+53} & 15 & 43 & 3.35 & BA 6 & +47 & 11 & 51 & 4.03 & $B A 6^{* *}$ \\
\hline & & & & & +58 & -55 & -11 & 4.12 & BA $20^{* * *}$ \\
\hline \multirow{2}{*}{-47} & -46 & -8 & 3.19 & BA 37* & -47 & -49 & -11 & 3.52 & BA $37^{*}$ \\
\hline & & & & & -38 & -55 & 47 & 3.14 & BA 40 \\
\hline+22 & -76 & 44 & 5.22 & $\mathrm{BA} 7^{* * *}$ & +22 & -76 & 44 & 6.38 & $\mathrm{BA} 7^{* * *}$ \\
\hline+28 & -74 & 41 & 4.41 & BA $19^{* * *}$ & +28 & -74 & 41 & 5.38 & BA $19^{* * *}$ \\
\hline+16 & -78 & 8 & 3.58 & BA $17^{* *}$ & +9 & -88 & 4 & 3.47 & BA $17^{* *}$ \\
\hline+12 & 16 & 3 & 3.63 & caudate $^{* *}$ & -9 & 1 & 11 & 3.18 & caudate $^{* *}$ \\
\hline \multicolumn{10}{|c|}{ Patients with schizophrenia > Healthy participants } \\
\hline-31 & 44 & 35 & 3.05 & BA 9 & +25 & 39 & 35 & 3.41 & BA 9 \\
\hline+34 & 39 & 42 & 4.08 & $\mathrm{BA} 8^{* *}$ & +31 & 39 & 39 & 5.01 & $\mathrm{BA} 8^{* * *}$ \\
\hline+6 & -9 & 59 & 3.67 & BA 6 & -3 & -15 & 60 & 4.16 & $B A 6^{* * *}$ \\
\hline+3 & 11 & 40 & 3.42 & BA $32^{* *}$ & +3 & 42 & -5 & 4.63 & BA $32^{* * *}$ \\
\hline-16 & -25 & 38 & 3.43 & BA $31^{*}$ & -3 & -44 & 32 & 3.22 & BA $31^{* *}$ \\
\hline \multirow[t]{3}{*}{68} & -21 & -2 & 3.64 & $\mathrm{BA} 21^{* *}$ & +41 & -4 & -27 & 3.51 & $\mathrm{BA} 21^{* *}$ \\
\hline & & & & & +31 & -34 & -12 & 4.01 & BA $36^{* * *}$ \\
\hline & & & & & -12 & -36 & 2 & 3.42 & $\mathrm{BA} 27^{* * *}$ \\
\hline \multirow[t]{4}{*}{-52} & -58 & 40 & 2.97 & BA 40 & -52 & -23 & 16 & 3.74 & BA $40^{*}$ \\
\hline & & & & & -16 & -43 & -1 & 4.11 & BA 19** \\
\hline & & & & & -6 & -97 & 12 & 3.82 & BA $18^{* *}$ \\
\hline & & & & & -16 & -33 & 9 & 3.57 & pulvinar** \\
\hline
\end{tabular}

*False discovery rate (FDR) corrected $p<0.08$; **FDR corrected $p<0.05$; ***FDR corrected $p<0.005$. Extent threshold $k=3$ voxels, voxel-level $p<0.005$, uncorrected.

learning. Although Murray et al. (2008) have shown normal probabilistic learning and performance in conjunction with reduced midbrain, striatum, hippocampus, and cingulate activation in first episode psychosis patients, they did not suggest how consistently less relative activation in these brain regions would translate to normal learning and performance in these patients. In the present study, matched comparison of good learners revealed greater activation of the cingulate, parahippocampal and parietal cortices in patients relative to healthy adults. This suggests that patients may successfully learn the probabilistic associations via parahippocampal or parietal cortex activation. In a sample of early stage, mildly affected Parkinson's disease patients, decreased caudate and increased medial temporal lobe activation was shown to yield normal probabilistic category learning and performance (Moody et al., 2004). Also, at least one type of implicit learning has recently been shown to activate hippocampus and influence explicit memory in healthy adults (Degonda et al., 2005). Evidence from non-human primate tracing studies has shown extensive connectivity between the inferior parietal cortex and the parahippocampal gyrus (Ding et al., 2000; Rozzi et al., 2006). A human diffusion weighted imaging study has also shown that the inferior parietal cortex and in particular the angular gyrus had a high probability of connectivity with the parahippocampal gyrus (Rushworth et al., 2006). Kircher et al. (2008) demonstrated a relationship between successful encoding/retrieval and parahippocampal-inferior parietal activation. Koch et al. (2008) has shown increased frontal-parietal activity under conditions of increasing uncertainty during probability learning. In the present study, patients also displayed greater activity in a more rostral region of dorsolateral prefrontal cortex (BA 9) that may have facilitated learning in these patients. Other prefrontal regions have been associated with good performance in patients with schizophrenia during working memory (Callicott et al.,
2003). Also, after initial dorsolateral prefrontal hyperactivation, patients with schizophrenia display a trend toward decreased activity along with decreased reaction times similar to healthy adults during overlearning of a working memory task (Koch et al., 2007). Regarding greater cingulate activity in patients, previous work has shown that deciding versus monitoring in probability-based reward environments was related to caudal and anterior cingulate activation, respectively in healthy adults (Behrens et al., 2007). Thus, greater cingulate activity in patients showing normal learning and performance may represent greater conflict monitoring and decision making.

There are other potential alternative explanations for the results obtained from the present study. Relative to healthy adults, patients with schizophrenia show greater variation in their neural activation patterns during a given cognitive task (MeyerLindenberg et al., 2002). Antipsychotic medications may also influence activation patterns displayed in these patients. All patients (except one) were receiving second generation antipsychotic medication which may have influenced neural activity particularly within the caudate relative to healthy participants. Previous studies (Hoptman et al., 2006; McClure et al., 2006; Glenthoj et al., 2007; Okugawa et al., 2007; van Haren et al., 2007; Goldman et al., 2008) have shown a relationship between antipsychotic administration and caudate nucleus volume, caudate hypometabolism in treatment resistant patients (Molina et al., 2007) and significantly lower relative glucose metabolism in the caudate (Buchsbaum et al., 2007) of patients with schizophrenia. However, caudate hypometabolism has also been shown in antipsychotic naive patients (Molina et al., 2007) and in first episode psychosis patients, in which 5 of 13 patients were not receiving antipsychotic medication, patients displayed diminished striatal activity with increasing uncertainty during probability learning (Murray et al., 2008). Domenger and Schwarting 
(2008) found that injections of 6-hydroxydopamine into the rodent striatum (which reduces striatal dopamine binding) produced impaired serial reaction time performance, and Fletcher (1995) showed that injections of 5,7dihydroxytryptamine into rodent median raphe nucleus reduced striatal 5-HT and 5-hydroxyindoleacetic acid levels and decreased performance on a differentialreinforcement-of-low-rate $20 \mathrm{~s}$ schedule while preserving rate of improvement over time, which is analogous behaviorally to what is observed in relation to probabilistic category learning of patients with schizophrenia who are treated with antipsychotic medication. Thus, dissociation of probabilistic category learning rate and performance in patients with schizophrenia relative to healthy participants may be a result of successful antipsychotic treatment; conversely, in the present study there appears to be very little to no effect of antipsychotic medication on probabilistic category learning based on the correlation between chlorpromazine equivalent dose and probabilistic category learning acquisition rate.

Although cognitive strategy analyses from the whole sample revealed potentially different cognitive strategies used by patients and healthy participants showing learning, the majority of patients and healthy adults showing learning during quartiles 2 and 3 used identical strategies and showed different patterns of brain activation to achieve successful probabilistic category learning. Thus, at least for the majority of participants during quartiles 2 and 3, strategy analysis differences alone would not sufficiently account for the differential brain activation displayed between patients with schizophrenia and healthy participants from the whole sample. Also, other strategy analysis variables (i.e., trial at which first strategy switch occurred, total number of strategy switches, number of strategy switches during the first two quartiles, and number of strategy switches during the last two quartiles) did not differ significantly between patients and healthy participants. In the matched comparison of good learners there was no significant difference between groups on the basis of strategies used.

In summary, patients with schizophrenia display a failure to sufficiently activate dorsolateral prefrontal cortex and caudate nucleus during probabilistic category learning. Relative to healthy participants classified as good learners, those patients with schizophrenia classified as good learners display differential activation of a more rostral region of dorsolateral prefrontal cortex, cingulate, parahippocampal gyrus, and inferior parietal cortex that may represent a compensatory mechanism in those patients who learn the probabilistic associations. These results confirm that probabilistic category learning can occur in the absence of normal frontal-striatal function. Overall, based on the stringent definition of learning used to differentiate good and poor learners, fewer patients with schizophrenia attain normal probabilistic category learning rates and performance levels relative to healthy adults. The impaired overall performance observed in the whole sample of patients with schizophrenia appears to be driven by a larger proportion of patients with abnormal learning rates.

\section{References}

Alexander GE, DeLong MR, Strick PL (1986) Parallel organization of functionally segregated circuits linking basal ganglia and cortex. Annu Rev Neurosci 9:357-381.

Behrens TE, Woolrich MW, Walton ME, Rushworth MF (2007) Learning the value of information in an uncertain world. Nat Neurosci 10:1214-1221.

Bertolino A, Knable MB, Saunders RC, Callicott JH, Kolachana B, Mattay VS, Bachevalier J, Frank JA, Egan M, Weinberger DR (1999) The relationship between dorsolateral prefrontal $N$-acetylaspartate mea- 
sures and striatal dopamine activity in schizophrenia. Biol Psychiatry 45:660-667.

Bertolino A, Breier A, Callicott JH, Adler C, Mattay VS, Shapiro M, Frank JA, Pickar D, Weinberger DR (2000) The relationship between dorsolateral prefrontal neuronal $\mathrm{N}$-acetylaspartate and evoked release of striatal dopamine in schizophrenia. Neuropsychopharmacology 22:125-132.

Buchsbaum MS, Buchsbaum BR, Hazlett EA, Haznedar MM, Newmark R, Tang CY, Hof PR (2007) Relative glucose metabolic rate higher in white matter in patients with schizophrenia. Am J Psychiatry 164:1072-1081.

Callicott JH, Mattay VS, Verchinski BA, Marenco S, Egan MF, Weinberger DR (2003) Complexity of prefrontal cortical dysfunction in schizophrenia: more than up or down. Am J Psychiatry 160:2209-2215.

Clare L, McKenna PJ, Mortimer AM, Baddeley AD (1993) Memory in schizophrenia: what is impaired and what is preserved? Neuropsychologia 31:1225-1241.

Curtis VA, Dixon TA, Morris RG, Bullmore ET, Brammer MJ, Williams SC, Sharma T, Murray RM, McGuire PK (2001) Differential frontal activation in schizophrenia and bipolar illness during verbal fluency. J Affect Disord 66:111-121.

Davis SW, Dennis NA, Daselaar SM, Fleck MS, Cabeza R (2008) Qué PASA? The posterior-anterior shift in aging. Cereb Cortex 18:1201-1209.

Degonda N, Mondadori CR, Bosshardt S, Schmidt CF, Boesiger P, Nitsch RM, Hock C, Henke K (2005) Implicit associative learning engages the hippocampus and interacts with explicit associative learning. Neuron 46:505-520.

Ding SL, Van Hoesen G, Rockland KS (2000) Inferior parietal lobule projections to the presubiculum and neighboring ventromedial temporal cortical areas. J Comp Neurol 425:510-530.

Domenger D, Schwarting RKW (2008) Effects of neostriatal 6-OHDA lesion on performance in a rat sequential reaction time task. Neurosci Lett 444:212-216.

Eyler LT, Jeste DV, Brown GG (2008) Brain response abnormalities during verbal learning among patients with schizophrenia. Psychiatry Res 162:11-25.

Fletcher PJ (1995) Effects of combined or separate 5,7dihydroxytryptamine lesions of the dorsal and median raphe nuclei on responding maintained by a DRL 20s schedule of reinforcement. Brain Res 675:45-54.

Fera F, Weickert TW, Goldberg TE, Tessitore A, Hariri A, Das S, Lee S, Zoltick B, Meeter M, Myers CE, Gluck MA, Weinberger DR, Mattay VS (2005) Neural mechanisms underlying probabilistic category learning in normal aging. J Neurosci 25:11340-11348.

Foerde K, Poldrack RA, Knowlton BJ, Sabb FW, Bookheimer SY, Bilder RM, Guthrie D, Granholm E, Nuechterlein KH, Marder SR, Asarnow RF (2008) Selective cortical dysfunction in schizophrenia: examination of motor and cognitive skill leraning. Neuropsychology 22:100-109.

Genovese CR, Lazar NA, Nichols T (2002) Thresholding of statistical maps in functional neuroimaging using the false discovery rate. Neuroimage 15:870-878.

Glenthoj A, Glenthoj BY, Mackeprang T, Pagsberg AK, Hemmingsen RP, Jernigan TL, Baaré WF (2007) Basal ganglia volumes in drug-naïve, first-episode schizophrenia patients before and after short-term treatment with either a typical or an atypical antipsychotic drug. Psychiatry Res 154:199-208.

Gluck MA, Shohamy D, Myers C (2002) How do people solve the "weather prediction" task?: individual variability in strategies for probabilistic category learning. Learn Mem 9:408-418.

Goldman AL, Pezawas L, Mattay VS, Fischl B, Verchinski BA, Zoltick B, Weinberger DR, Meyer-Lindenberg A (2008) Heritability of brain morphology related to schizophrenia: a large-scale, automated magnetic resonance imaging segmentation study. Biol Psychiatry 63:475-483.

Gould RL, Brown RG, Owen AM, Bullmore ET, Howard RJ (2006) Taskinduced deactivations during successful paired associates learning: An effect of age but not Alzheimer's disease. Neuroimage 31:818-831.

Heinz A, Knable MB, Coppola R, Gorey JG, Jones DW, Lee KS, Weinberger DR (1998) Psychomotor slowing, negative symptoms and dopamine receptor availability-an IBZM SPECT study in neuroleptic-treated and drug-free schizophrenic patients. Schizophr Res 31:19-26.

Hoptman MJ, Volavka J, Czobor P, Gerig G, Chakos M, Blocher J, Citrome
LL, Sheitman B, Lindenmayer JP, Lieberman JA, Bilder RM (2006) Aggression and quantitative MRI measures of caudate in patients with chronic schizophrenia or schizoaffective disorder. J Neuropsychiatry Clin Neurosci 28:509-515.

Howard DV, Howard JH Jr (1989) Age differences in learning serial patterns: Direct versus indirect measures. Psychol Aging 4:357-364.

Joel D, Zohar O, Afek M, Hermesh H, Lerner L, Kuperman R, Gross-Isseroff R, Weizman A, Inzelberg R (2005) Impaired procedural learning in obsessive-compulsive disorder and Parkinson's disease, not in major depressive disorder. Behav Brain Res 157:253-263.

Johnston DW, Bursill AE (1973) Probability learning and width of attention in normal and schizophrenic groups. Br J Soc Clin Psychol 12:402-410.

Kay SR, Fiszbein A, Opler LA (1987) The positive and negative syndrome scale (PANSS) for schizophrenia. Schizophr Bull 13:261-276.

Kéri S, Kelemen O, Szekeres G, Bagóczky N, Erdélyi R, Antal A, Benedek G, Janka Z (2000) Schizophrenics know more than they can tell: probabilistic classification learning in schizophrenia. Psychol Med 30:149-155.

Kéri S, Juhász A, Rimanóczy A, Szekeres G, Kelemen O, Cimmer C, Szendi I, Benedek G, Janka Z (2005) Habit learning and the genetics of the dopamine D3 receptor: evidence from patients with schizophrenia and healthy controls. Behav Neurosci 119:687-693.

Kircher T, Weis S, Leube D, Freymann K, Erb M, Jessen F, Grodd W, Heun R, Krach S (2008) Anterior hippocampus orchestrates successful encoding and retrieval of non-relational memory: an event-related fMRI study. Eur Arch Psychiatry Clin Neurosci 258:363-372.

Koch K, Wagner G, Nenadic I, Schachtzabel C, Roebel M, Schultz C, Axer M, Reichenbach JR, Sauer H, Schlösser RG (2007) Temporal modeling demonstrates preserved overlearning processes in schizophrenia: an fMRI study. Neuroscience 146:1474-1483.

Koch K, Schachtzabel C, Wagner G, Reichenbach JR, Sauer H, Schlösser R (2008) The neural correlates of reward-related trial-and-error learning: an fMRI study with a probabilistic learning task. Learn Mem 15:728-732.

Knowlton BJ, Squire LR, Gluck MA (1994) Probabilistic classification learning in amnesia. Learn Mem 1:106-120.

Knowlton BJ, Mangels JA, Squire LR (1996a) A neostriatal habit learning system in humans. Science 273:1399-1402.

Knowlton BJ, Squire LR, Paulsen JS, Swerdlow NR, Swenson M, Butters N (1996b) Dissociations with non-declarative memory in Huntington's disease. Neuropsychology 10:538-548.

McClure RK, Phillips I, Jazayerli R, Barnett A, Coppola R, Weinberger DR (2006) Regional change in brain morphometry in schizophrenia associated with antipsychotic treatment. Psychiatry Res 148:121-132.

Meeter M, Myers CE, Shohamy D, Hopkins RO, Gluck MA (2006) Strategies in probabilistic categorization: results from a new way of analyzing performance. Learn Mem 13:230-239.

Meeter M, Radics G, Myers CE, Gluck MA, Hopkins RO (2008) Probabilistic categorization: how do normal participants and amnesic patients do it? Neurosci Biobehav Rev 32:237-248.

Menon V, Aldeman NE, White CD, Glover GH, Reiss AL (2001) Errorrelated brain activation during a go/no-go response inhibition task. Hum Brain Mapp 12:131-143.

Meyer-Lindenberg A, Miletich RS, Kohn PD, Esposito G, Carson RE, Quarantelli M, Weinberger DR, Berman KF (2002) Reduced prefrontal activity predicts exaggerated striatal dopaminergic function in schizophrenia. Nat Neurosci 5:267-271.

Molina V, Sanz J, Sarramea F, Palomo T (2007) Marked hypofrontality in clozapine-responsive patients. Pharmacopsychiatry 40:157-162.

Moody TD, Bookheimer SY, Vanek Z, Knowlton BJ (2004) An implicit learning task activates medial temporal lobe in patient's with Parkinson's disease. Behav Neurosci 118:438-442.

Murray GK, Corlett PR, Clark L, Pessiglione M, Blackwell AD, Honey G, Jones PB, Bullmore ET, Robbins TW, Fletcher PC (2008) Substantia nigra/ventral tegmental reward prediction error disruption in psychosis. Mol Psychiatry 13:267-276.

Mutter SA, Pliske RM (1994) Aging and illusory correlation in judgments of co-occurrence. Psychol Aging 9:53-63.

Okugawa G, Nobuhara K, Takase K, Saito Y, Yoshimura M, Kinoshita T (2007) Olanzapine increases grey and white matter volumes in the caudate nucleus of patients with schizophrenia. Neuropsychobiology 55:43-46.

Pickar D, Su TP, Weinberger DR, Coppola R, Malhotra AK, Knable MB, Lee 
KS, Gorey J, Bartko JJ, Breier A, Hsiao J (1996) Individual variation in D2 dopamine receptor occupancy in clozapine-treated patients. Am J Psychiatry 153:1571-1578.

Poldrack RA, Prabhakaran V, Seger CA, Gabrieli JD (1999) Striatal activation during acquisition of a cognitive skill. Neuropsychology 13:564-574.

Poldrack RA, Clark J, Paré-Blagoev EJ, Shohamy D, Creso Moyano J, Myers C, Gluck MA (2001) Interactive memory systems in the human brain. Nature 414:546-550.

Reiss JP, Campbell DW, Leslie WD, Paulus MP, Ryner LN, Polimeni JO, Foot BJ, Sareen J (2006) Deficit in schizophrenia to recruit the striatum in implicit learning: a functional magnetic resonance imaging investigation. Schizophr Res 87:127-137.

Rozzi S, Calzavara R, Belmalih A, Borra E, Gregoriou GG, Matelli M, Luppino G (2006) Cortical connections of the inferior parietal cortical convexity of the macaque monkey. Cereb Cortex 16:1389-1417.

Rushworth MF, Behrens TE, Johansen-Berg H (2006) Connection patterns distinguish three regions of human parietal cortex. Cereb Cortex $16: 1418-1430$

Schönberg T, Daw ND, Joel D, O’Doherty JP (2007) Reinforcement learning signals in the human striatum distinguish learners from nonlearners during reward-based decision making. J Neurosci 27:12860-12867.

Tan HY, Callicott JH, Weinberger DR (2007) Dysfunctional and compensatory prefrontal cortical systems, genes and the pathogenesis of schizophrenia. Cereb Cortex 17 (Suppl 1):i171-i181.

van Haren NE, Hulshoff Pol HE, Schnack HG, Cahn W, Mandl RC, Collins DL, Evans AC, Kahn RS (2007) Focal gray matter changes in schizophrenia across the course of the illness: a 5-year follow-up study. Neuropsychopharmacology 32:2057-2066.

Weickert TW, Terrazas A, Bigelow LB, Malley JD, Hyde T, Egan MF, Weinberger DR, Goldberg TE (2002) Habit and skill learning in schizophrenia: evidence of normal striatal processing with abnormal cortical input. Learn Mem 9:430-442. 